\title{
The effect of taping on maximum plantar pressure and ground reaction force in people with flat foot after applying a fatigue protocol
}

Fatemeh Aghakeshizadeh

Kharazmi University

Amir Letafatkar

Kharazmi University

Peyman Aghaei Ataabadi

Kharazmi University

Mahdi Hosseinzadeh ( $\nabla$ metti@ssrc.ac.ir)

Sport Sciences Research Institute

\section{Research Article}

Keywords: taping, plantar pressure, ground reaction force, fatigue, flat foot.

Posted Date: May 7th, 2021

DOl: https://doi.org/10.21203/rs.3.rs-492886/v1

License: (c) (1) This work is licensed under a Creative Commons Attribution 4.0 International License.

Read Full License 


\section{Abstract}

Background: People suffering from flat foot show more movements in hindfoot and midfoot joints as compared to the others. The anti-pronation tapings are supposed to provide temporary external support for the medial longitudinal arch. The aim of this study was to examine the effects of two types of antipronation taping on the lower limb kinetics in flat foot people before and after performing a physical fatigue protocol.

Methods: 20 male and female with flat foot aged $22.39 \pm 2.02$ years old were studied under three conditions (untaping, reverse-6 taping and low-dye taping) either before or after fatigue states. The maximum plantar pressure and ground reaction force were measured by an RSscan foot scan system during walking.

Results: A statistically significant difference was observed after applying two types of taping (reverse-6 vs. low-dye taping) in the maximum plantar pressure perceived in metatarsus $1(P=0.016)$ and lateral heel $(P=0.044)$. In the post-fatigue conditions, there were significant differences between the two taping types in metatarsus $4(P=0.024)$. The maximum ground reaction force in toe $1(P=0.001)$, toe $2-5(P=$ $0.001)$, metatarsus $5(P=0.001)$, and medial heel $(P=0.001)$ was significantly different between reverse6 and Low-dye tapings.

Conclusions: The results indicated that the low-dye and reverse- 6 taping types can reduce the pressure on the medial side of the foot, and push it towards the lateral side. It is therefore suggested using taping as an effective treatment for redistribution of the pressure and force in sole of the foot in people with flat foot.

\section{Background}

The foot structure is considered as an important body segment which is responsible for shock absorbing, forces transferring, body propulsioning, and stabilizing the body during different activities (1). These functions are possible due to an existing biomechanical relationship among transverse arch, medial longitudinal arch and lateral longitudinal arch of the foot. Unnatural foot arch changes may cause functional changes in the foot structure. Caused by unnatural decrease or flattening or loss of the medial longitudinal arch (2). Flat foot is considered as one of the most prevalent injuries of the lower extremity $(3,4)$. In fact, people suffering from flat foot show more movements in hindfoot and midfoot joints as compared to the others (5), and their foot remains pronated for a longer period of time during the gait cycle (6).

This condition has been demonstrated as contributing factor to many lower limb overuse injuries so that researchers have indicated that excessive foot pronation may be associated with reduction of body stability (7), medial tibial stress syndrome (8), plantar fasciitis (9), and patellofemoral pain syndrome (8). It is also suggested that excessive foot pronation can make the foot and leg muscles more susceptible to be fatigable (10). As a further matter, this postural deformity, as excessed pronation, alters joint kinetics, 
which finally changes joints loading and muscle activation (11). Following reduction or loss of the longitudinal arch, the plantar pressure distribution is disturbed or interrupted; forces are therefore applied on deeper tissues and upper joints, leading in conditions such as pain in these joints $(12,13)$. Such an improper distribution of plantar forces causes abnormal movements, and leads to apply stress in the lower limb structures, and finally it effectively develops muscle dysfunctions (14). It therefore seems that individuals with pronated foot are more exposed or vulnerable to the injury risk as compared to the healthy ones (5). It is actually, required to take necessary actions to prevent and treat this abnormality in order to possibly minimize its consequences.

As a new mostly-used method, plantar pressure measurements are used to quantify static and dynamic pressures as a measure of foot and ankle function during gait (15); since the foot and ankle provide both the necessary support and flexibility for weight bearing and weight shifting while performing these activities, such data collection can help therapists managing the impairments associated with various musculoskeletal and neurological disorders (16).

Furthermore, fatigue which is considered as one of the main factors to alter biomechanical parameters, causes an increase in the domain of postural fluctuations, a decrease in the ability to maintain balance, disturbance in proprioception (17), plantar pressure distribution (18) and changes other kinetic and kinematic parameters of the body joints (19). Headlee et al. (2008) investigated the effect of fatigue of the plantar intrinsic muscles of the foot on the foot arch height. They reported an increase in navicular drop under fatigue conditions. Accordingly, fatigue was considered as a significant factor for changing the medial longitudinal arch (20), which can affect the interventional treatments.

Various treatments have been introduced to correct and treat excessive foot pronation, including orthoses (21), medical insoles (22), taping and orthopedic interventions (23). However, as in sports conditions, when a person cannot use suitable shoes or as it is not possible to use medical insoles, it seems a good alternative to use taping. Taping has recently been significantly used to prevent and treat musculoskeletal disorders, especially for foot disorders (18). The anti-pronation tapings are supposed to provide temporary external support for the medial longitudinal arch (24), maintain the shape and height of the arch (24), reduce the midtarsal joint movements, and decrease the pressure imposed upon the medial foot part (6).

The effects of various forms of taping have relatively been explored on motor performance, lower- limb mechanics and sensory inputs in several studies $(25,26)$; of such different taping forms, low-dye and reverse- 6 tapings have received the greatest attention among researchers. By using low-dye taping (made of inelastic tapes), the range of motion and the foot muscle activity can be respectively limited and adjusted $(27,28)$. Evidence suggests that low-dye taping can cause the plantar pressure distribution to be altered and corrected by shifting the pressure of the midfoot from the inside to the outside, and altering the hindfoot and forefoot forces through reducing the pressure in the forefoot and increasing that in the hindfoot and midfoot parts $(6,28-30)$. Moreover, reverse- 6 taping technique therefore traverses both malleoli and has been advocated to control excess foot pronation (31). According to previous studies, 
applying reverse- 6 taping technique limits the range of the talocrural joint motion, especially in the sagittal plane (plantar flexion) (32). In a meta-analysis study conducted by Cheung et al. (2012), it was indicated that reverse- 6 taping applied to the proximal part of the talocrural joint is more effective in controlling foot pronation as compared with low-dye taping.

Conducted studies and existing evidence indicate that low-dye taping makes the plantar pressure and force distribution modulated in people with flat foot $(6,28,30,33)$. However, to the best knowledge of the authors no research has yet been performed to investigate the effect of reverse- 6 taping on the plantar pressure. Given such lack of information, most researches have examined the instant effects of taping on plantar pressure. The present study was therefore intended to study the effects of two anti-pronation taping models on the maximum plantar pressure and ground reaction force distribution related to individuals with flat foot before and after a period of fatigue. It was assumed in this research that applying the foot arch taping helps to correct the plantar pressure and force distribution, and these effects become more prominent in the fatigue conditions.

\section{Methods}

\section{Design}

A Quasi-experimental study with pretest - posttest design was conducted to evaluate the effects of two anti-pronation taping models on the maximum plantar pressure and ground reaction force distribution related to individuals with flat foot before and after a period of fatigue.

\section{Participants}

Twenty flat footed individuals (male and female) participated in this study. The age (mean \pm standard deviation), height, mass, and foot size of the participants respectively were $22.4 \pm 2.0$ years, $171 \pm 6.8 \mathrm{~cm}$, $71.4 \pm 8.4 \mathrm{~kg}$, and $43.3 \pm 2.6$. An a priori sample size estimate using an $a=0.05$, a $\beta=0.20$, a withinfactor correlation of 0.7 , and a partial eta squared effect size statistic of 0.06 (moderate effect size) was conducted using G*Power software (Version 3.1, University of Dusseldorf, Dusseldorf, Germany) (30, 3335). This sample size estimate indicated that a minimum of 20 subjects was needed in order to ensure adequate power considering our planned statistical analysis (repeated measures analysis of variance).

The protocol of the study was approved by the Institutional Review Board at [omitted for review]. Before enrollment volunteered athletes were informed of the risks associated with the study, and signed an institutionally approved consent document to perform the tests and the procedures of the study.

Inclusion criteria consisted of people with navicular drop greater than $10 \mathrm{~mm}$ (6) and being able to walk pain free (30). Exclusion criteria included any sensitivity or abnormal reaction to rigid taping, such as redness, having rash or discomfort $(6,30)$, lower limb injuries in the past six months $(6,30)$, surgery on the lower limb (33), and neurological disorders (33). Before measuring the variables, a summary of the research design was explained to the subjects. They were then initially screened based on the research 
inclusion and exclusion criteria, and those who were voluntarily willing to cooperate completed the consent form. Having met the inclusion criteria, the included subjects were let to enter the laboratory for performing the testing process based on the previously announced time. On the test day, their background information including height, weight, and age were recorded in the demographic data collection form.

\section{Initial Subjective Interview}

The subjects' demographic information, disease details and duration if applied, medical history and related injuries were determined through personal interviews with the subjects. The interviews were conducted by a researcher with more than ten years of experiences in assessing and rehabilitating musculoskeletal disorders.

Maximum plantar pressure and ground reaction force were recorded while walking in three different conditions (untaping, low-dye taping and reverse-6 taping) as well as in two time points, before and after fatigue; the data collection has been randomly conducted through three different days with one-week interval.

Prior to each measurement session, the participants were first familiar with the laboratory environment, and the testing procedures e.g. walking, and/or stepping at their desired speed in the designated path. Five walking repetitions were recorded on the foot-scan device in both the pre-and post-fatigue conditions, along with applying each of three intended taping conditions (untaping, low-dye taping and reverse-6 taping). In this study, it was tried to control all the testing conditions, including testing time points, conditions, fatigue and other factors affecting the results so that the testing conditions were similarly performed in all three days.

\section{Clinical Assessments}

How to record maximum plantar pressure variables and maximum ground reaction force: Foot-scan device (RsScan international, Belgium, 40-100 cm, 8192 sensors, $253 \mathrm{~Hz}$ ) was used to record the variables related to plantar pressure and force distribution during walking; foot-scan 7 gait generation software was then used to analyze the obtained data. The foot-scan device was located in the center of a 12-meter-long path to record individuals' natural gait. To record the participants' plantar pressure and force distribution, they were asked to walk in a certain direction at their normal speed, and walk over the foot-scan. To avoid changes in the step length, they were asked to go back and forth several times, and were asked not to change their gait pattern or not to adjust their steps before going on the foot-scan so that their second step was exactly felling on the foot-scan. Each gait test was repeated three times, and a one minute rest interval between two gait recordings was considered. In order to analyze the studied variables, foot-scan 7 gait generation software automatically divided the foot sole into ten anatomical areas including first toe (Toe 1), second to fifth toes (Toe 2-5), first to fifth metatarsal heads (Meta1-5), midfoot, medial heel, and lateral heel. Then, the dependent variables of maximum plantar pressure and force respectively in terms of $\mathrm{N} / \mathrm{cm}^{2}$ and $\mathrm{N}$ were recorded in the ten foot sole areas using the Foot-scan device (RsScan International, Belgium, 40 _ 100 cm, 8192 sensors, $253 \mathrm{~Hz}$ ) (35). 


\section{Procedure}

Low-dye taping: the low-dye taping technique consisted of a rigid and sticky white LP tape (LP athletic tape comprises of $100 \%$ cotton/zinc oxide professional grade athletic tape. Width: $38 \mathrm{~mm}$, company: LP support UK). In order to apply the taping, the participants' ankle was placed in a neutral position. Longitudinal anchor strips were pulled lateral-to-medial along the lateral aspect of the foot starting at the head of the fifth metatarsal and ending at the head of the first metatarsal. Then, the transverse arch support strip was pulled lateral-to-medial along the plantar surface of the foot starting at the anterior surface of the calcaneus to the heads of the metatarsals. Finally, the taping was completed with an additional longitudinal anchor strip applied to help secure the transverse arch support strips (36).

Reverse-6 Taping: reverse-6, which its effect has already been shown to increase the navicular height, included a LP strip (LP athletic tape comprises of 100\% cotton/zinc oxide professional grade athletic tape. Width: $38 \mathrm{~mm}$, company: LP support UK), started from the medial malleolus and coursed anterolaterally over the foot, and then, it continued from under the midfoot up to the medial side of the foot and distal leg to support the mid and rear-foot. The ankle and foot were placed in a slight supination position in order to effectively apply the taping (37).

Fatigue protocol: The fatigue protocol included running on a treadmill. Having familiarized the participants with the test conditions and the fatigue protocol, each participant began to walk on a treadmill at a speed of $6-\mathrm{km} / \mathrm{h}$. Participants were asked to report perceived exertion according to the 15point Borg Scale while walking on treadmill (6-20). Furthermore, participants' heart rate was simultaneously monitored. Speed was increased in increments of $1 \mathrm{~km} / \mathrm{h}$ every 2 min until an intensity of 13 on the Borg scale was reached. After reporting a score of 13 , subjects continued to run at the given steady state speed until a Borg score of 17 or $80 \%$ of their age related heart rate peak was reached. As reached this very point, they continued to run for 2 additional minutes. Then, the participants were immediately tested for walking on the foot scan to evaluate the effect of foot taping on the research variables in the fatigue condition. Finally, the participants performed the cool-down protocol at a selfselected speed. As a further matter, in order to equalize the test conditions for all the participants and to prevent the bias in this regard, they were all provided with a new neutral running shoes for the runninginduced fatigue protocol (35).

\section{Statistical analysis}

Descriptive statistics was used to describe the data (mean and standard deviation) for all the variables and participants' demographic information. Shapiro-Wilk test was also used to measure normality of data distribution. A 3*2 factorial ANOVA (three groups consisting of control vs. revers- 6 vs. low dye taping * time points consisting of pre vs. post fatigue) was used to compare the taping and fatigue-caused main effects, and their interactive effects. If the variance analysis results were significant, a Bonferroni post hoc test was used to compare taping levels between different treatments. All the statistical comparisons were performed using SPSS software version 20, at a significance level set at $\leq 0.05$. 


\section{Results}

\section{Toe1}

A significant main effect of Time ${ }^{\star}$ Group interaction was observed for Toe1 maximum plantar pressure ( $F$ $(2,57)=26.907, \mathrm{P}=0.001)$.

Comparing the pre-post within-subject, the untaped condition $(P=0.001)$ and Low-dye taping $(P=0.007)$ showed a significant decrease, however Reverse- 6 taping showed a significant increase $(P=0.001)$ in Toe1 maximum plantar pressure after fatigue.

The between subject analysis for Toe1 maximum plantar pressure revealed a strong tendency in the difference between untaped condition and Reverse- 6 taping $(P=0.033)$ at the pre fatigue; so that Toe1 maximum plantar pressure after Reverse-6 taping was significantly lower than untaped condition.

\section{Toe 2-5}

A significant main effect of Time was observed for Toe2-5 maximum plantar pressure $(F(1,57)=11.274$; $P=0.001)$. A Time ${ }^{\star}$ Group interaction was also detected for Toe2-5 maximum plantar pressure $(F$ $(2,57)=6.914 ; p=0.026)$.

Comparing the pre-post within-subject, the untaped condition $(P=0.029)$ and Low-dye taping $(P=0.001)$ showed a significant decrease in Toe2-5 maximum plantar pressure after fatigue. Maximum plantar pressure did not significantly change after fatigue in the Reverse-6 taping.

The between-subject analysis for Toe2-5 maximum plantar pressure did not show any significant difference between groups at the pre and/or post-fatigue time points $(p=0.05)$.

\section{Meta1}

A significant main effect of Time was observed for Meta1 maximum plantar pressure $(F(1,57)=33.263$; $P=0.001)$.

Comparing the pre-post within-subject, the untaped condition $(P=0.006)$, Low-dye taping $(P=0.014)$ and Reverse-6 taping $(P=0.001)$ showed a significant increase in Meta1 maximum plantar pressure after fatigue.

The between-subject analysis for Meta1 maximum plantar pressure did not show any significant difference between groups at the pre and/or post-fatigue conditions $(p=0.05)$.

\section{Meta2}

A significant main effect of Time was observed for Meta2 maximum plantar pressure $(F(1,57)=12.561$; $P=0.001)$. A main effect of Group was detected for Meta2 maximum plantar pressure $(F(2,57)=7.311 ; p=$ 0.001 ). 
Comparing the pre-post within-subject, the untaped condition $(P=0.007)$ and Low-dye taping $(P=0.004)$ showed a significant decrease in Meta2 maximum plantar pressure after fatigue.

The between subject analysis for Meta2 maximum plantar pressure revealed a strong tendency in the difference between untaped condition and Reverse- 6 taping ( $P=0.007)$; and between Reverse- 6 and Lowdye taping $(P=0.016)$ at the pre fatigue time point; so that Meta2 maximum plantar pressure via applying Reverse- 6 taping was significantly lower than Low-dye taping and untaped condition. After fatigue the between subject analysis revealed a strong tendency in the difference between untaped condition and Reverse- 6 taping $(P=0.016)$; so that Meta2 maximum plantar pressure after Reverse- 6 taping was significantly lower than untaped condition.

\section{Meta3}

A significant main effect of Time was observed for Meta3 maximum plantar pressure $(F(1,57)=4.387 ; P$ $=0.041)$.

Comparing the pre-post within-subject, the untaped condition $(P=0.027)$ showed a significant increase in Meta3 maximum plantar pressure after fatigue. Maximum plantar pressure did not significantly change after fatigue in the Reverse- 6 and Low-dye taping condition.

The between-subject analysis for Meta3 did not show any significant difference between groups at the pre-fatigue $(p=0.05)$. After fatigue the between subject analysis revealed a strong tendency in the difference between untaped condition and Reverse- 6 taping $(P=0.046)$; so that Meta3 maximum plantar pressure after Reverse-6 taping was significantly lower than untaped condition.

\section{Meta4}

A significant main effect of Time*Group interaction was detected for Meta4 maximum plantar pressure ( $F$ $(2,57)=3.512 ; p=0.036)$.

Comparing the pre-post within-subject, the Reverse- 6 taping $(P=0.046)$ showed a significant decrease in Meta4 maximum plantar pressure after fatigue. Maximum plantar pressure did not significantly change after fatigue in the Low-dye taping and untaped condition.

The between-subject analysis for Meta4 did not show a significant difference between groups at the prefatigue ( $p=0.05)$. After fatigue the between subject analysis revealed a strong tendency in the difference between Low-dye and Reverse- 6 taping $(P=0.024)$; so that Meta4 maximum plantar pressure after Reverse-6 taping was significantly lower than after applying Low-dye taping.

\section{Medial heel}

A significant main effect of Time was observed for Medial heel maximum plantar pressure $(F(1,57)=$ 4.214; $\mathrm{P}=0.045)$. A Time ${ }^{\star}$ Group interaction was also detected for Medial heel maximum plantar pressure $(F(2,57)=7.906 ; p=0.001)$. 
Comparing the pre-post within-subject, the Reverse- 6 taping $(P=0.001)$ showed a significant increase in Medial heel maximum plantar pressure after fatigue. Maximum plantar pressure did not significantly change after fatigue in the Low-dye taping and untaped condition.

The between-subject analysis for Medial heel maximum plantar pressure did not show any significant difference between groups at the pre and post-fatigue $(p=0.05)$.

\section{Lateral heel}

A main effect of Group was observed for Lateral heel maximum plantar pressure $(F(2,57)=4.310 ; p=$ 0.018).

Comparing the pre-post within-subject, did not show any significant change in Lateral heel maximum plantar pressure for Reverse- 6 and Low-dye taping and untapped condition after fatigue.

The between subject analysis for Lateral heel maximum plantar pressure revealed a strong tendency in the difference between Low-dye and Reverse- 6 taping $(P=0.044)$ at the pre fatigue; so that Lateral heel maximum plantar pressure at Low-dye taping was significantly lower than Reverse- 6 taping. After fatigue the between subject analysis revealed a strong tendency in the difference between untaped condition and Low-dye taping $(P=0.041)$; so that Lateral heel maximum plantar pressure after Low-dye taping was significantly lower than untaped condition.

\section{Maximum ground reaction force}

\section{Toe1}

A significant main effect of Time was observed for Toe1 maximum force $(F(1,57)=26.681 ; P=0.001)$. A Time $^{\star}$ Group interaction was also detected for Toe1 maximum force $(F(2,57)=75.812 ; p=0.001)$.

Comparing the pre-post within-subject, the Reverse- 6 taping $(P=0.001)$ showed significant increase and Low-dye taping $(P=0.001)$ showed a significant decrease in Toe1 maximum force after fatigue.

The between subject analysis for Toe 1 maximum force revealed a strong tendency in the difference between untaped condition and Low-dye taping $(P=0.001)$; and between Reverse- 6 and Low-dye taping $(P=0.001)$ at the pre fatigue; so that Toe1 maximum force at Reverse- 6 taping and untaped condition were significantly lower than Low-dye taping. After fatigue the between subject analysis revealed a strong tendency in the difference between untapped condition and Reverse- 6 taping $(P=0.001)$, untapped condition and Low-dye taping ( $P=0.002)$; and Reverse- 6 and Low-dye taping $(P=0.001)$; so that Toe1 maximum force at Low-dye taping was significantly lower than untapped condition, and untapped condition was significantly lower than Reverse-6 taping.

\section{Toe2-5}


A significant main effect of Time ${ }^{\star}$ Group interaction was detected for Toe2-5 maximum force ( $F$ $(2,57)=8.161 ; p=0.001)$.

Comparing the pre-post within-subject, the Reverse- 6 taping $(P=0.023)$ showed significant increase and Low-dye taping $(P=0.001)$ showed a significant decrease in Toe2-5 maximum force after fatigue.

The between subject analysis for Toe2-5 maximum force revealed a strong tendency in the difference between untapped condition and Reverse- 6 taping $(P=0.002)$, and Reverse- 6 and Low-dye taping $(P=$ 0.001 ) at the pre fatigue; so that Toe2-5 maximum force at Reverse- 6 taping was significantly lower than Low-dye taping and untapped condition. The between-subject analysis for Toe2-5 did not show any significant difference between groups at the post-fatigue condition $(p=0.05)$.

\section{Meta1}

A significant main effect of Time was observed for Meta1 maximum force $(F(1,57)=22.781 ; P=0.001)$. A Time*Group interaction was detected for Meta1 maximum force $(F(2,57)=6.297 ; p=0.003)$.

Comparing the pre-post within-subject, the Low-dye taping $(P=0.001)$ showed a significant increase in Meta1 maximum force after fatigue.

The between-subject analysis for Meta 1 maximum force did not show any significant difference between groups at the pre and post-fatigue conditions $(p=0.05)$.

\section{Meta2}

A significant main effect of Time was observed for Meta2 maximum force $(F(1,57)=13.108 ; P=0.001)$. A Time* Group interaction was detected for Meta2 maximum force $(F(2,57)=15.117 ; p=0.001)$.

Comparing the pre-post within-subject, the Reverse-6 $(P=0.023)$ and Low-dye taping $(P=0.034)$ showed a significant decrease in Meta2 maximum force after fatigue.

The between-subject analysis for Meta2 did not show any significant difference between groups at the pre-fatigue time point $(p=0.05)$. The between subject analysis for Meta2 maximum force revealed a strong tendency in the difference between untapped condition and Reverse- 6 taping $(P=0.010)$ at the post fatigue time point; so that Meta 2 maximum force after Reverse- 6 taping was significantly lower than untapped condition.

\section{Meta3}

A significant main effect of Time was observed for Meta3 maximum force $(F(1,57)=7.924 ; P=0.007)$. Comparing the pre-post within-subject, the Low-dye taping $(P=0.005)$ showed a significant decrease in Meta3 maximum force after fatigue. 
The between-subject analysis for Meta3 maximum force did not show any significant difference between groups at the pre and post-fatigue time point $(p=0.05)$.

\section{Meta4}

A significant main effect of Time*Group interaction was detected for Meta4 maximum force $(F(2,57)=$ $3.606 ; p=0.034)$.

Comparing the pre-post within-subject, the Reverse-6 taping $(P=0.041)$ showed a significant decrease in Meta4 maximum force after fatigue.

The between-subject analysis for Meta 4 did not show any significant difference between groups at the pre-fatigue time point $(p=0.05)$. The between subject analysis for Meta4 maximum force revealed a strong tendency in the difference between Low-dye and Reverse- 6 taping $(P=0.006)$ at the post fatigue time point; so that Meta4 maximum force after Reverse- 6 taping was significantly lower than Low-dye taping.

\section{Meta5}

A significant main effect of Time was observed for Meta5 maximum force $(F(1,57)=17.852 ; P=0.001)$. A Time*Group interaction was also detected for Meta5 maximum force $(F(2,57)=16.660 ; p=0.001)$. A significant main effect of Group was further observed for Meta5 maximum force $(F(2,57)=30.702 ; P=$ $0.001)$.

Comparing the pre-post within-subject, the Reverse- 6 taping $(P=0.027)$ showed significant decrease, and Low-dye taping $(P=0.001)$ and untapped condition $(P=0.001)$ showed a significant increase in Meta5 maximum force after fatigue.

The between subject analysis for Meta5 maximum force revealed a strong tendency in the difference between untapped condition and Low-dye taping $(P=0.001)$, and Reverse- 6 and Low-dye taping $(P=$ 0.001) at the pre fatigue time point; so that Meta5 maximum force after Low-dye taping was significantly higher than Reverse- 6 taping and untapped condition. After fatigue the between subject analysis revealed a strong tendency in the difference between untapped condition and Reverse- 6 taping $(P=0.014)$, untapped condition and Low-dye taping $(P=0.001)$, and Reverse- 6 and Low-dye taping $(P=0.001)$; so that Meta5 maximum force at Reverse- 6 taping was significantly lower than untapped condition and untapped condition was significantly lower than Low-dye taping.

\section{Medial heel}

A significant main effect of Time ${ }^{\star}$ Group interaction was detected for Medial heel maximum force ( $F$ $(2,57)=10.118 ; p=0.001)$. A significant main effect of Group was also observed for Medial heel maximum force $(F(2,57)=15.676 ; P=0.001)$. 
Comparing the pre-post within-subject, the Reverse- 6 taping $(P=0.001)$ showed a significant increase in Medial heel maximum force after fatigue.

The between subject analysis for Medial heel maximum force revealed a strong tendency in the difference between untapped condition and Low-dye taping $(P=0.026)$, untapped condition and Reverse6 taping $(P=0.001)$, and Reverse- 6 and Low-dye taping $(P=0.001)$ at the pre fatigue time point; so that Medial heel maximum force at Reverse- 6 taping was significantly lower than Low-dye taping, and Lowdye taping was significantly lower than untapped condition. After fatigue the between subject analysis revealed a strong tendency in the difference between untaped condition and Low-dye taping $(P=0.040)$; so that Medial heel maximum force after Low-dye taping was significantly lower than untaped condition.

\section{Lateral heel}

A significant main effect of Time ${ }^{\star}$ Group interaction was detected for Lateral heel maximum force ( $F$ $(2,57)=6.671 ; p=0.002)$. A significant main effect of Group was also observed for Lateral heel maximum force $(F(2,57)=8.353 ; P=0.001)$.

Comparing the pre-post within-subject, the Low-dye taping $(P=0.007)$ showed a significant decrease and untaped condition $(P=0.007)$ showed significant increase in Lateral heel maximum force after fatigue.

The between-subject analysis for Lateral heel did not show any significant difference between groups at the pre-fatigue time point $(p=0.05)$. After fatigue the between subject analysis for Lateral heel maximum force revealed a strong tendency in the difference between untaped condition and Low-dye taping $(P=$ $0.001)$, and untaped condition and Reverse- 6 taping $(P=0.015)$; so that Lateral heel maximum force at Reverse- 6 and Low-dye taping were significantly lower than untaped condition. 
Table 1

Comparison of the parameters of maximum plantar pressure and force on the ten areas of the foot

\begin{tabular}{|c|c|c|c|c|c|}
\hline \multirow[t]{2}{*}{ Regions } & \multirow[t]{2}{*}{ Variables } & \multicolumn{3}{|c|}{ within-subjects effect } & \multirow{2}{*}{$\begin{array}{l}\text { Pairwise } \\
\text { comparisons } \\
\text { Bonferroni post } \\
\text { hoc test results } \\
\text { (post-fatigue) }\end{array}$} \\
\hline & & $\begin{array}{l}\text { tape } \times \text { Fatigue } \\
\text { interaction effect } \\
\omega\end{array}$ & $\begin{array}{l}\text { effects of } \\
\text { fatigue } \omega\end{array}$ & $\begin{array}{l}\text { effects of } \\
\text { taping } \omega\end{array}$ & \\
\hline \multirow[t]{2}{*}{ Toe1 } & pressure & $26.91(0.001)^{\star}$ & $2.36(0.130)$ & $0.69(0.504)$ & $\begin{array}{l}1.00 \Omega ; 0.860 ¥ ; \\
1.00 \dagger\end{array}$ \\
\hline & force & $75.81(0.001)^{\star}$ & $26.68(0.001) \star$ & $1.60(0.212)$ & $\begin{array}{l}0.002 \Omega ; 0.001 ¥ \\
0.001 \dagger\end{array}$ \\
\hline \multirow[t]{2}{*}{ Toe2-5 } & pressure & $3.878(0.026)^{\star}$ & $11.274(0.001) *$ & $0.21(0.979)$ & $\begin{array}{l}1.00 \Omega ; 1.00 ¥ ; \\
0.772 \dagger\end{array}$ \\
\hline & force & $8.16(0.001) \star$ & $0.48(0.493)$ & $1.92(0.155)$ & $\begin{array}{l}0.183 \Omega ; 1.00 ¥ ; \\
0.375 \dagger\end{array}$ \\
\hline \multirow[t]{2}{*}{ Meta1 } & pressure & $1.251(0.294)$ & $33.263(0.001) *$ & $0.079(0.925)$ & $\begin{array}{l}1.00 \Omega ; 1.00 ¥ ; \\
1.00 \dagger\end{array}$ \\
\hline & force & $6.30(0.003) \star$ & $22.78(0.001)^{\star}$ & $0.15(0.865)$ & $\begin{array}{l}0.450 \Omega ; 1.00 ¥ ; \\
0.933 \dagger\end{array}$ \\
\hline \multirow[t]{2}{*}{ Meta2 } & pressure & $2.14(0.128)$ & $12.56(0.001)^{*}$ & $7.31(0.001)^{\star}$ & $0.016 ¥$ \\
\hline & force & $15.12(0.001)^{\star}$ & $13.11(0.001)^{\star}$ & $1.66(0.199)$ & $0.010 ¥$ \\
\hline \multirow[t]{2}{*}{ Meta3 } & pressure & $1.21(0.305)$ & $4.39(0.041) *$ & $2.09(0.133)$ & $0.046 ¥$ \\
\hline & force & $1.26(0.291)$ & $7.92(0.007)$ * & $2.74(0.073)$ & $\begin{array}{l}0.074 \Omega ; 0.644 ¥ \\
0.887 \dagger\end{array}$ \\
\hline \multirow[t]{2}{*}{ Meta4 } & pressure & $3.51(0.036) *$ & $0.44(0.507)$ & $2.14(0.127)$ & $0.024 \dagger$ \\
\hline & force & $3.61(0.034) *$ & $0.02(0.886)$ & $3.04(0.056)$ & $0.006 \dagger$ \\
\hline \multirow[t]{2}{*}{ Meta5 } & pressure & $1.06(0.354)$ & $1.85(0.180)$ & $0.40(0.669)$ & $\begin{array}{l}1.00 \Omega ; 1.00 ¥ ; \\
1.00 \dagger\end{array}$ \\
\hline & force & $16.66(0.001)^{\star}$ & $17.85(0.001)^{\star}$ & $30.70(0.001)^{\star}$ & $\begin{array}{l}0.001 \Omega ; 0.014 ¥ \\
0.001 \dagger\end{array}$ \\
\hline \multirow[t]{2}{*}{ Midfoot } & pressure & $0.40(0.673)$ & $0.53(0.467)$ & $0.02(0.980)$ & $\begin{array}{l}1.00 \Omega ; 1.00 ¥ ; \\
1.00 \dagger\end{array}$ \\
\hline & force & $1.22(0.304)$ & $1.35(0.250)$ & $0.26(0.773)$ & $\begin{array}{l}1.00 \Omega ; 1.00 ¥ ; \\
1.00 \dagger\end{array}$ \\
\hline \multirow[t]{2}{*}{ Medialheel } & pressure & $7.91(0.001) *$ & $4.21(0.045)^{\star}$ & $0.10(0.908)$ & $\begin{array}{l}1.00 \Omega ; 1.00 ¥ ; \\
0.725 \dagger\end{array}$ \\
\hline & force & $10.12(0.001)^{\star}$ & $2.10(0.089)$ & $15.68(0.001)^{\star}$ & $0.040 \Omega$ \\
\hline
\end{tabular}




\begin{tabular}{|c|c|c|c|c|c|}
\hline \multirow[t]{2}{*}{ Regions } & \multirow[t]{2}{*}{ Variables } & \multicolumn{3}{|c|}{ within-subjects effect } & \multirow{2}{*}{$\begin{array}{l}\text { Pairwise } \\
\text { comparisons } \\
\text { Bonferroni post } \\
\text { hoc test results } \\
\text { (post-fatigue) }\end{array}$} \\
\hline & & $\begin{array}{l}\text { tape } \times \text { Fatigue } \\
\text { interaction effect } \\
\omega\end{array}$ & $\begin{array}{l}\text { effects of } \\
\text { fatigue } \omega\end{array}$ & $\begin{array}{l}\text { effects of } \\
\text { taping } \omega\end{array}$ & \\
\hline \multirow[t]{2}{*}{ Lateralheel } & pressure & $0.51(0.601)$ & $2.03(0.159)$ & $4.31(0.018)^{\star}$ & $0.041 \Omega$ \\
\hline & force & $6.67(0.002)^{\star}$ & $1.91(0.172)$ & $8.35(0.001)^{\star}$ & $0.001 \Omega ; 0.015 ¥$ \\
\hline \multicolumn{6}{|c|}{$\Omega$ Significant between Low-dye taping and untapped. } \\
\hline \multicolumn{6}{|c|}{$¥$ Significant between reverse- 6 taping and untapped. } \\
\hline \multicolumn{6}{|c|}{ † Significant between reverse- 6 taping and Low-dye taping. } \\
\hline \multicolumn{6}{|c|}{ * Significant of 2 * 3 analysis of variance test results } \\
\hline \multicolumn{6}{|c|}{$\omega$ Values stands for $F(P-V a l u e)}$. \\
\hline
\end{tabular}

\section{Discussion}

This study was aimed to investigate the effects of two types of anti-pronation taping on maximum plantar pressure and ground reaction force distribution in people with flat foot, before and after applying the fatigable protocol.

\section{Maximum plantar pressure pre-fatigue}

Having applied the Reverse- 6 taping, the obtained results indicated that flat footed participants showed significant decrease at Toe1 $(P=0.033)$ and Meta2 $(P=0.007)$ as compared to the untaping condition and Low-dye taping with Meta2 $(P=0.016)$. In Lateral heel $(P=0.044)$ Low-dye taping showed significant decrease compared to Reverse-6 taping.

\section{Maximum plantar pressure post-fatigue}

As for Meta2 $(P=0.016)$ and Meta3 $(P=0.046)$, the Reverse-6 taping induced significant decrease compared to untaping condition. In Meta4 $(P=0.024)$ Reverse- 6 taping led to significant decrease as compared to Low-dye taping. In Lateral heel $(P=0.041)$ Low-dye taping led to significant decrease compared to untaping condition.

\section{Ground reaction force pre-fatigue}

In Toe1 Reverse- 6 taping $(P=0.001)$ and untaping condition $(P=0.001)$ showed significant decrease compared to Low-dye taping. In Toe2-5 Reverse-6 taping showed significant decrease compared to Lowdye taping $(P=0.001)$ and untaping condition $(P=0.002)$. In Meta5 Low-dye taping showed significant 
increase compared to Reverse- 6 taping $(P=0.001)$ and untaping condition $(P=0.001)$. In Medial heel Reverse-6 taping $(P=0.001)$ and Low-dye taping $(P=0.026)$ showed significant decrease compared to untaping condition $(P=0.001)$, and Reverse- 6 taping $(P=0.001)$ decrease compared to Low-dye taping.

\section{Ground reaction force post-fatigue}

In Toe1 Reverse-6 taping showed significant increase compared to Low-dye taping $(P=0.001)$ and untaping condition ( $P=0.001)$; and Low-dye taping $(P=0.002)$ decrease compared to untaping condition. In Meta2 Reverse- 6 taping showed significant decrease compared to untaping condition $(P=0.002)$. In Meta4 Reverse- 6 taping showed significant decrease compared to Low-dye taping $(P=0.001)$. In Meta5 Low-dye taping showed significant increase compared to Reverse- 6 taping $(P=0.001)$ and untaping condition $(P=0.001)$, and Reverse-6 taping $(P=0.014)$ decrease compared to untaping condition. In Medial heel Low-dye taping $(P=0.026)$ showed significant decrease compared to untaping condition $(P=$ 0.040). In Lateral heel Reverse- 6 taping $(P=0.015)$ and Low-dye taping $(P=0.001)$ decrease compared to untaping condition.

The findings of the present study emphasize and highlight importance of appropriate management of forces and pressures imposed on the foot joints based on the patient symptoms which should be considered by physicians and exercise specialists to reduce them, and to empower patients using due strategies in managing joint movements. These results should not disappoint physicians to persistently try in favor of prescribing definitive treatments for flat foot. Rather, the findings obtained from the present study emphasize the role of temporarily interventions to minimize the effects caused by flat foot during long-term activities.

Lange et al. (2004) stated that Low-Dye taping significantly increases peak and mean plantar pressure in the lateral subpart of the midfoot and under the toes, and it decreases the pressure under the heel and forefoot, indicating a reduction in foot pronation. Applying Low-Dye taping in the present study significantly reduced the pressure under the heel and the medial and middle forefoot, while increasing pressure under the lateral midfoot and under the toes. Moreover, a significant decrease was observed in the mean plantar pressure on the lateral forefoot, as no significant difference was observed in the peak plantar pressure in this area. However, there was no significant change in peak or mean pressure under the medial midfoot (6).

Newel (2015) revealed that taping techniques have a greater effect on redistributing plantar pressures. Specifically, as taping could effectively shift pressures laterally, pressures on the outside of the foot were hereby increased. On the other side, it successfully decreases forefoot plantar pressures. In addition, these researchers stated that plantar pressure seems to be a clinically important measurement. Therefore, quite a more attention should be paid to how the plantar pressure distribution is changed by taping foot $\operatorname{arch}(24)$.

Taping can alter the level of pressure and generally the load on the heel, either by restricting pronation of the subtalar and midtarsal joints, or via changing the proprioception in the heel. Tapping induced- 
decreased pressure under the lateral and medial heel may lead to transferring more weight to the lateral midfoot. This may be due to the restriction of the subtalar and midtarsal joints pronation and facilitation activity of the tibialis posterior, which is the principal supinator of the foot (6).

Furthermore, using transverse strips may restrict the foot movements. This can cause more pressure on the lateral midfoot. The transverse strips were intended to provide a strong supination force to support the medial longitudinal arch, and restrict pronation (28). This increase in the plantar pressure can further be due to a decrease in the forefoot pressure. If the foot was more stable, stretching the digital flexor directly and extensor tendons may increase the efficiency of the toes in the propulsive stance phase. Hence, the pressure would be transferred forward onto the toes, and pressure is applied by the toes instead of the forefoot (6).

Nolan and Kennedy (2009) observed that the plantar pressures in the medial and lateral areas of the forefoot began to return to the pre-taping level following a 10-minute walking, and this pattern will be kept for next 20 minutes. However, the plantar pressure tendency to shift medially to laterally in the midfoot was maintained within 20 minutes (38).

Quinn et al. (2010) showed that, during exercise activities, including cross-cut, side-cut, and shuttle run, the maximum force in the medial and lateral midfoot area increases in people with flat foot, and this leads to a high risk of injuries in the medial and lateral midfoot (39). According to a study conducted by Park and Kim (2019), during natural walking, the maximum forces at the medial rear-foot and big toe were higher in athletes with exercise-induced leg pain than in those without leg pain, and peak pressures on the lateral midfoot and toes were more in athletes with exercise-induced leg pain compared to those without the leg pain. Based on the findings of the upper mentioned study, the anti-pronation taping technique only altered the peak pressure of the medial midfoot during natural walking. It seems that their research results are related to a very short-term period of adaptation time using the anti-pronation taping (33).

Following exercise, Lee et al. (2012) demonstrated that the medial forefoot and the medial midfoot show a significant difference in the plantar pressure. In the medial forefoot, the pressure increased from 21.83 to 25.95. Also, it increased from 5.52 to 12.75 in the medial midfoot. Increased subtalar joint pronation was observed in people with flat foot after fatigue appeared in the plantar intrinsic muscles (34).

It seems that the plantar intrinsic muscles play an important role in supporting the dynamic arch, implying such muscles support the excessive foot arch drop during walking. In this regard, when the plantar intrinsic muscles function is completely lost, the navicular drop is significantly increased along with the tibial nerve block, which can entirely indicate the loss of the medial longitudinal arch (its integrity) (40). This is in line with the findings of Headlee (2008) where impaired function of the plantar intrinsic muscles negatively affects the structural integrity of the medial longitudinal arch in the static stance disregarding the initial navicural drop. These results indicate that the plantar intrinsic muscles, regardless of the arch height measurement, provide the medial longitudinal arch with essential support in the static stance, increasing individuals' foot pronation after fatigue (20). 
The results of the current study in line with the mentioned studies indicates $\mathrm{X}$ or $\mathrm{Z}$ taping can be used trying to neutralize the malfunction and adverse effect of flat foot induced plantar pressure and force distribution both before and after the foot muscle fatigue.

\section{Conclusions}

Taping the foot arches can modify the kinetics of the lower limbs in people with flat foot after a period of fatigue. Both low-dye and reverse- 6 tapings can improve the distribution of maximum plantar pressure and force, and they, to some extent, are able recover the plantar pressure and force to the untaping status after fatigue comparing to untaping condition. Taping can specifically decrease the pressure and force through the medial part of the foot and increase them through the lateral part of the foot in flat-footed people. They can therefore be suggested to people with flat foot. According to the results obtained from the present study, it is suggested that applying taping might be considered as an effective temporarily treatment in combination with other treatment methods so as to rehabilitate people with flat foot during sports activities and daily life.

\section{Declarations}

\section{Ethics approval and consent to participate:}

The protocol of the study was approved by the Institutional Review Board at Kharazmi university, Tehran, Iran. The study was conducted in accordance with the Declaration of Helsinki and the International Committee of Medical Journal Editors after approval by the Ethics Committee in Research of Sport Sciences Research Institute of Iran (IR.SSRC.REC.1399.129). Before enrollment volunteered athletes were informed of the risks associated with the study, and then the signed informed consent document was obtained from all the participants. Participants had the right to withdraw from the study at any time without any consequences.

Consent for publication: Not applicable

Availability of data and materials: The datasets used and/or analysed during the current study are available from the corresponding author on reasonable request.

Competing interests: The authors declare that they have no competing interests.

Authors' contributions: FA and AL conceived and designed the research. FA conducted the experiments. $\mathrm{FA}, \mathrm{PAA}$, and $\mathrm{MH}$ analyzed and interpreted the data regarding the maximum plantar pressure and ground reaction force in the flat footed participants of this study. AL was a major contributor in writing the manuscript. All authors read and approved the final manuscript.

Acknowledgements: Not applicable. 


\section{References}

1. Rogério FRPG, dos Santos DJ, Desiderio AFS, de Freitas ERFS. Acute effect of low-Dye taping on dynamic plantar pressure in subjects with overpronation foot. Manual Therapy, Posturology \& Rehabilitation Journal. 2016:1-8.

2. FAPTA DANPP. Kinesiology of the Musculoskeletal System: Foundations for Rehabilitation. 2010.

3. Mirbagheri SS, Kashani RV, Mehraban F, Gharebaghy S. Prevalence of flat foot and hallux valgus deformity among primary school female students in Kiar city of Chaharmahal and Bakhtiari. Pajouhan Scientific Journal. 2013;11(4):36-41.

4. Kamali M, Janmohammadi M, Kamali A. Prevalence of flat foot in elementary, guidance and high school students (Babol 2005-2006). Journal of Babol University of Medical Sciences. 2008;9(6):50-4.

5. Aguilar MB, Abián-Vicén J, Halstead J, Gijon-Nogueron G. Effectiveness of neuromuscular taping on pronated foot posture and walking plantar pressures in amateur runners. J Sci Med Sport. 2016;19(4):348-53.

6. Lange B, Chipchase L, Evans A. The effect of low-Dye taping on plantar pressures, during gait, in subjects with navicular drop exceeding $10 \mathrm{~mm}$. J Orthop Sports Phys Ther. 2004;34(4):201-9.

7. Williams lii DS, McClay IS, Hamill J. Arch structure and injury patterns in runners. Clinical biomechanics. 2001;16(4):341-7.

8. Neal BS, Griffiths IB, Dowling GJ, Murley GS, Munteanu SE, Smith MMF, et al. Foot posture as a risk factor for lower limb overuse injury: a systematic review and meta-analysis. Journal of foot and ankle research. 2014;7(1):55.

9. Graham ME, Jawrani NT, Goel VK. Evaluating plantar fascia strain in hyperpronating cadaveric feet following an extra-osseous talotarsal stabilization procedure. The Journal of foot and ankle surgery. 2011;50(6):682-6.

10. Gray EG, Basmajian JV. Electromyography and cinematography of leg and foot ("normal" and flat) during walking. The anatomical record. 1968;161(1):1-15.

11. Kelly LA, Racinais S, Tanner CM, Grantham J, Chalabi H. Augmented low dye taping changes muscle activation patterns and plantar pressure during treadmill running. J Orthop Sports Phys Ther. 2010;40(10):648-55.

12. Lee MS, Vanore JV, Thomas JL, Catanzariti AR, Kogler G, Kravitz SR, et al. Diagnosis and treatment of adult flatfoot. The Journal of Foot and Ankle Surgery. 2005;44(2):78-113.

13. Chuckpaiwong B, Nunley JA, Mall NA, Queen RM. The effect of foot type on in-shoe plantar pressure during walking and running. Gait Posture. 2008;28(3):405-11.

14. Kwon O-Y, Mueller MJ. Walking patterns used to reduce forefoot plantar pressures in people with diabetic neuropathies. Phys Ther. 2001;81(2):828-35.

15. Firth J, Turner D, Smith W, Woodburn J, Helliwell P. The validity and reliability of PressureStat ${ }^{\text {TM }}$ for measuring plantar foot pressures in patients with rheumatoid arthritis. Clinical Biomechanics. 2007;22(5):603-6. 
16. Orlin MN, McPoil TG. Plantar pressure assessment. Phys Ther. 2000;80(4):399-409.

17. Harkins KM, Mattacola CG, UhI TL, Malone TR, McCrory JL. Effects of 2 ankle fatigue models on the duration of postural stability dysfunction. Journal of athletic training. 2005;40(3):191.

18. Williams S, Whatman C, Hume PA, Sheerin K. Kinesio taping in treatment and prevention of sports injuries. Sports Med. 2012;42(2):153-64.

19. Boozari S, Jamshidi AA, Sanjari MA, Jafari $H$. Effect of functional fatigue on vertical ground-reaction force in individuals with flat feet. Journal of sport rehabilitation. 2013;22(3):177-83.

20. Headlee DL, Leonard JL, Hart JM, Ingersoll CD, Hertel J. Fatigue of the plantar intrinsic foot muscles increases navicular drop. J Electromyogr Kinesiol. 2008;18(3):420-5.

21. Jafarnezhadgero AA, Shad MM, Majlesi M. Effect of foot orthoses on the medial longitudinal arch in children with flexible flatfoot deformity: A three-dimensional moment analysis. Gait Posture. 2017;55:75-80.

22. Hohmann E, Reaburn P, Tetsworth K, Imhoff A. Plantar pressures during long distance running: an investigation of 10 marathon runners. J Sports Sci Med. 2016;15(2):254.

23. Pinto RZ, Souza TR, Maher CG. External devices (including orthotics) to control excessive foot pronation. Br J Sports Med. 2012;46(2):110-1.

24. Newell T, Simon J, Docherty CL. Arch-taping techniques for altering navicular height and plantar pressures during activity. Journal of athletic training. 2015;50(8):825-32.

25. van de Water AT, Speksnijder CM. Efficacy of taping for the treatment of plantar fasciosis: a systematic review of controlled trials. J Am Podiatr Med Assoc. 2010;100(1):41-51.

26. Aminaka N, Gribble PA. A systematic review of the effects of therapeutic taping on patellofemoral pain syndrome. Journal of Athletic training. 2005;40(4):341.

27. Halseth T, McChesney JW, DeBeliso M, Vaughn R, Lien J. The effects of kinesio ${ }^{\mathrm{TM}}$ taping on proprioception at the ankle. J Sports Sci Med. 2004;3(1):1.

28. Russo SJ, Chipchase LS. The effect of low-Dye taping on peak plantar pressures of normal feet during gait. Aust J Physiother. 2001;47(4):239-44.

29. Carter K, Chockalingam N. An assessment of strapping techniques commonly used for pronated foot deformities. J Am Podiatr Med Assoc. 2009;99(5):391-8.

30. O'Sullivan K, Kennedy N, O'Neill E, Mhainin UN. The effect of low-dye taping on rearfoot motion and plantar pressure during the stance phase of gait. BMC Musculoskelet Disord. 2008;9(1):1-9.

31. Cheung RT, Chung RC, Ng GY. Efficacies of different external controls for excessive foot pronation: a meta-analysis. Br J Sports Med. 2011;45(9):743-51.

32. Delahunt E, O'Driscoll J, Moran K. Effects of taping and exercise on ankle joint movement in subjects with chronic ankle instability: a preliminary investigation. Arch Phys Med Rehabil. 2009;90(8):141822.

33. Park J, Kim T. Acute effect of taping on plantar pressure characteristics in athletes with exerciseinduced leg pain: a description and comparison of groups. The Physician and sportsmedicine. 
2019;47(2):212-9.

34. Lee C-R, Kim M-K, Cho MS. The relationship between balance and foot pressure in fatigue of the plantar intrinsic foot muscles of adults with flexible flatfoot. Journal of Physical Therapy Science. 2012;24(8):699-701.

35. Anbarian M, Esmaeili $\mathrm{H}$. Effects of running-induced fatigue on plantar pressure distribution in novice runners with different foot types. Gait Posture. 2016;48:52-6.

36. Yoho R, Rivera JJ, Renschler R, Vardaxis VG, Dikis J. A biomechanical analysis of the effects of lowDye taping on arch deformation during gait. The foot. 2012;22(4):283-6.

37. Cornwall MW, McPoil TG, Fair A. THE EFFECT OF EXERCISE AND TIME ON THE HEIGHT AND WIDTH OF THE MEDIAL LONGITUDINAL ARCH FOLLOWING THE MODIFIED REVERSE-6 AND THE MODIFIED AUGMENTED LOW-DYE TAPING PROCEDURES. Int J Sports Phys Ther. 2014;9(5):635.

38. Nolan D, Kennedy N. Effects of low-dye taping on plantar pressure pre and post exercise: an exploratory study. BMC Musculoskelet Disord. 2009;10(1):40.

39. Queen RM, Abbey AN, Wiegerinck JI, Yoder JC, Nunley JA. Effect of shoe type on plantar pressure: a gender comparison. Gait Posture. 2010;31(1):18-22.

40. Fiolkowski P, Brunt D, Bishop M, Woo R, Horodyski M. Intrinsic pedal musculature support of the medial longitudinal arch: an electromyography study. The Journal of foot and ankle surgery. 2003;42(6):327-33.

\section{Figures}

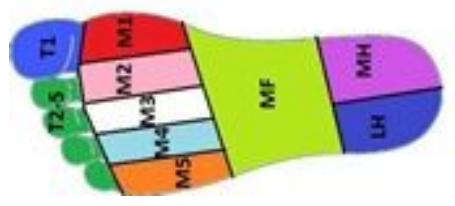

Figure 1

Division of the ten foot areas. Abbreviations: $\mathrm{T} 1=\mathrm{Toe} 1, \mathrm{~T} 2-5=\mathrm{Toe} 2-5, \mathrm{M} 1=$ Metatarsal1, M2=Metatarsal2, M3= Metatarsal3, M4= Metatarsal4, M5= Metatarsal5, MF=Midfoot, MH=Medialheel, LH=Lateralheel.
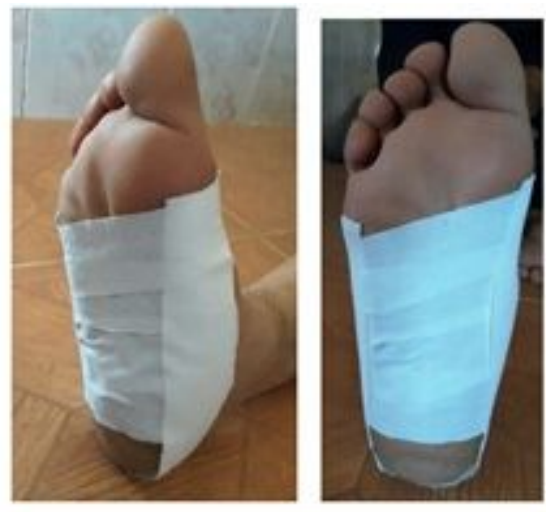

a b
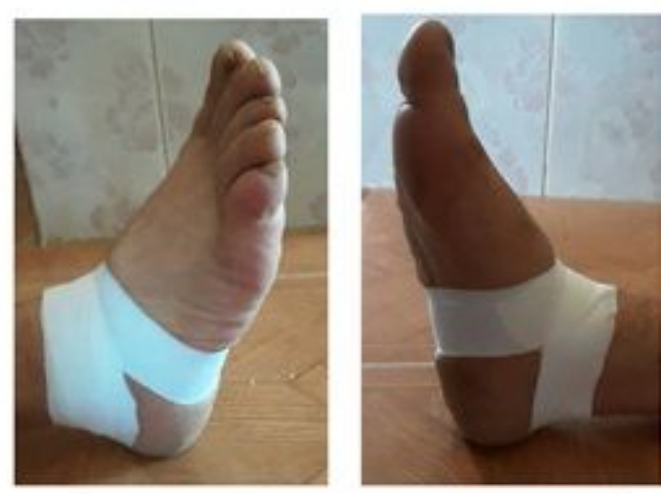

c d 
Figure 2

Low-dye taping (a-b) and Reverse taping (c-d) techniques

without Tape

\section{Figure 3}

Maximum plantar pressure between pre- and post-fatigue in each of the three measurement conditions. * Statistically significant difference comparing to pre test of the same intervention group.

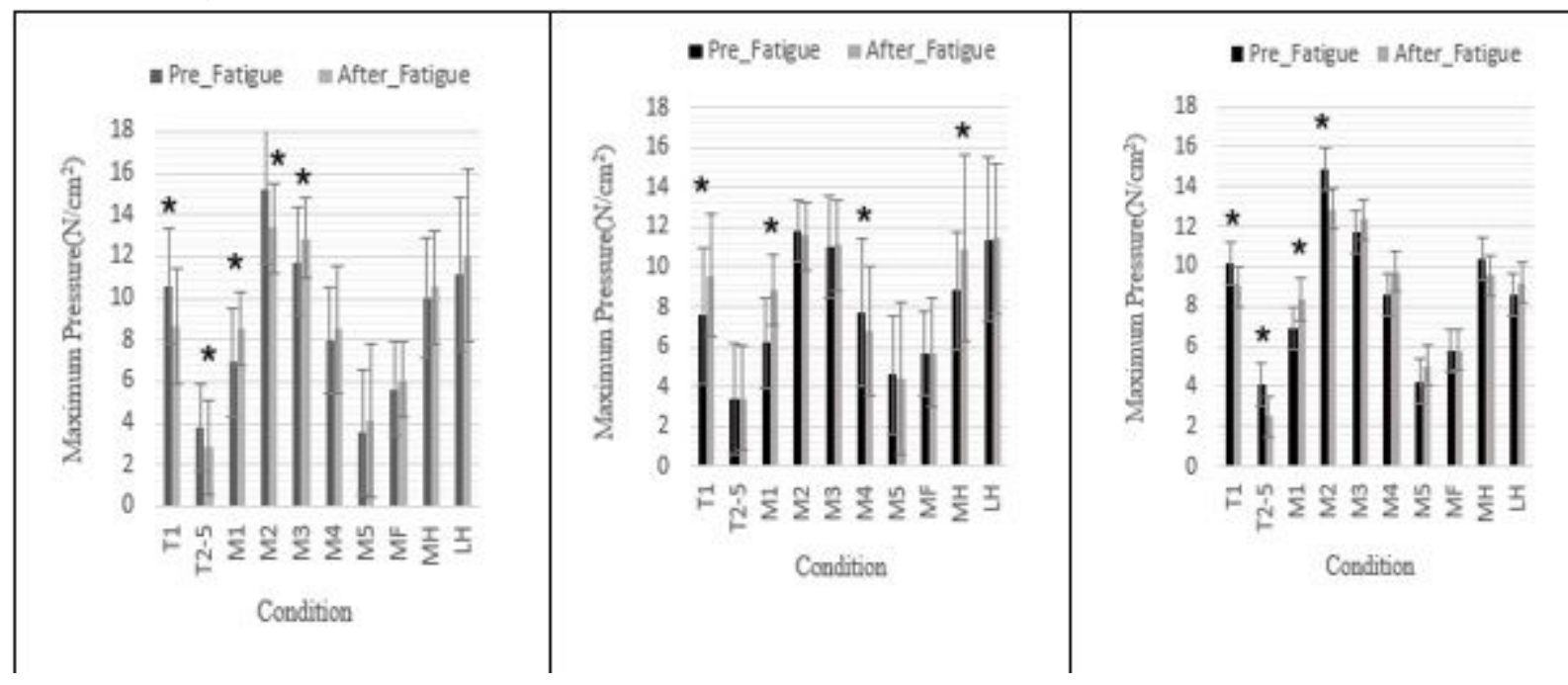

\section{Figure 4}

(mean \pm standard deviation) of the maximum plantar pressure before and after fatigue. * Statistically significant difference. Abbreviations: $\mathrm{T} 1=\mathrm{Toe} 1, \mathrm{~T} 2-5=\mathrm{Toe} 2-5, \mathrm{M} 1=$ Metatarsal1, M2=Metatarsal2, M3= Metatarsal3, M4= Metatarsal4, M5= Metatarsal5, MF=Midfoot, MH=Medialheel, LH=Lateralheel. 


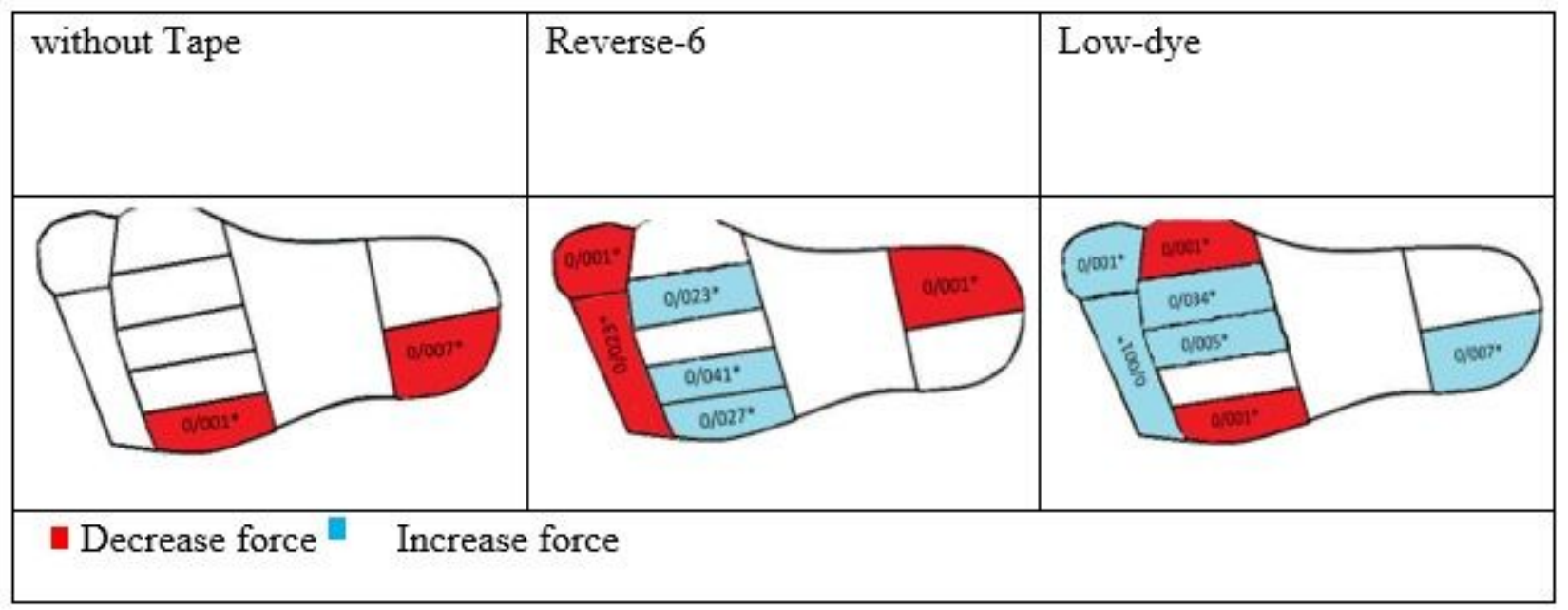

\section{Figure 5}

Maximum ground reaction force between pre- and post-fatigue in each of the three measurement conditions. * Statistically significant difference comparing to pretest of the same intervention group.
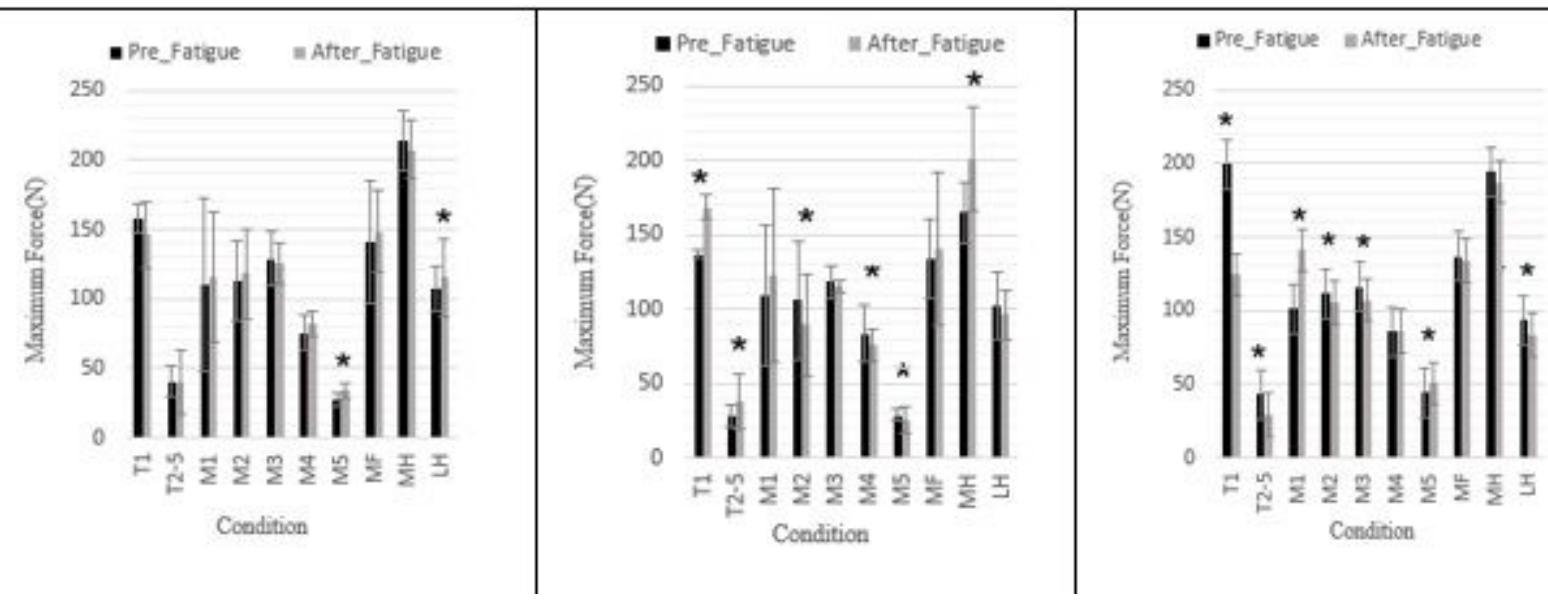

Figure 6

( mean \pm standard deviation) of the maximum ground reaction force before and after fatigue. * Statistically significan difference. Abbreviations: T1=Toe1, T2-5=Toe2-5, M1=Metatarsal1, M2=Metatarsal2, M3= Metatarsal3, M4= Metatarsal4, M5= Metatarsal5, MF=Midfoot, MH=Medialheel, $\mathrm{LH}=$ Lateralheel. 

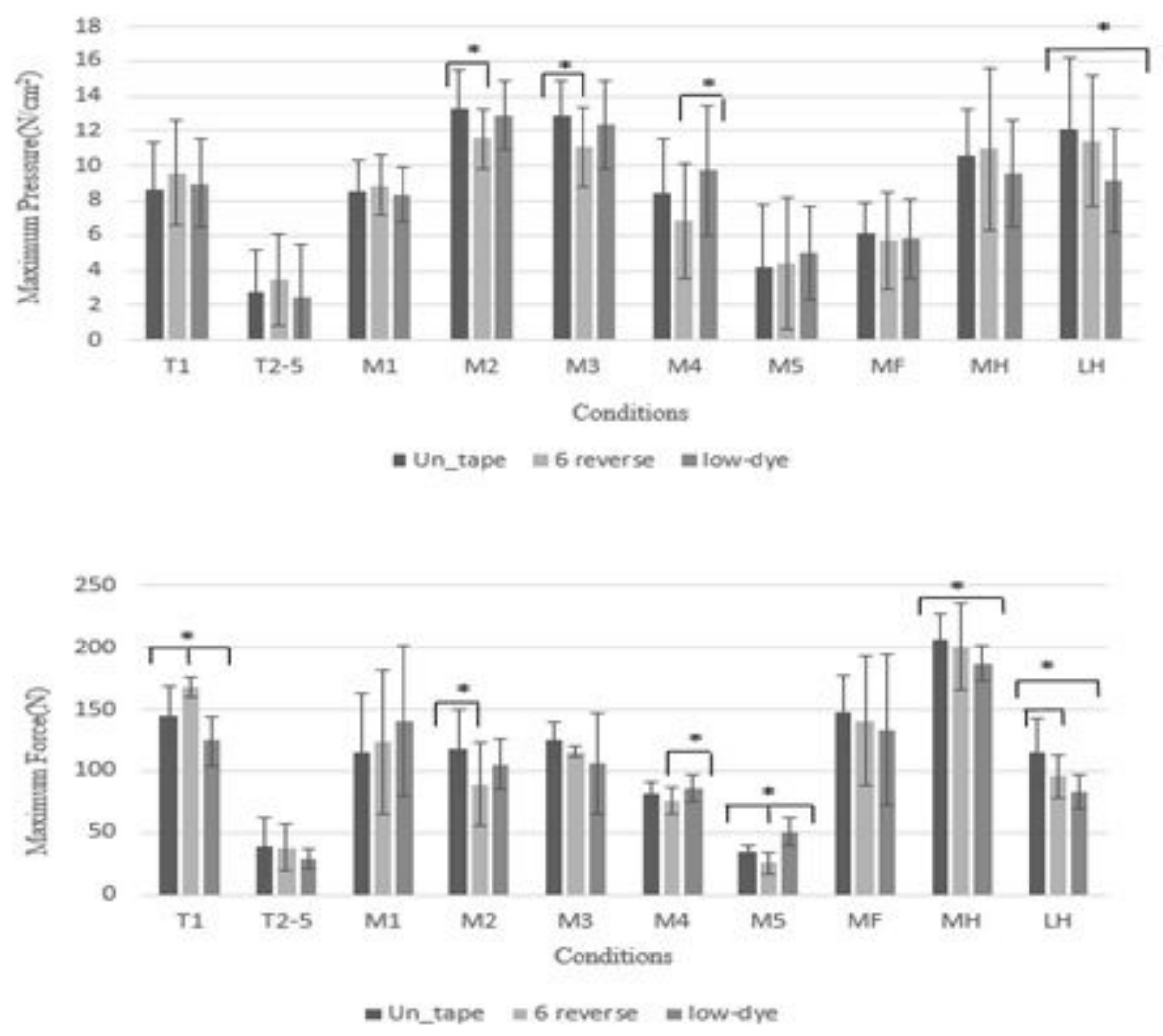

Figure 7

Between-group differences in the maximum plantar pressure and force after fatigue (analysis of variance with repeated measures). * Statistically significan difference. Abbreviations: T1=Toe1, T2-5=Toe2-5, M1 =Metatarsal1, M2=Metatarsal2, M3= Metatarsal3, M4= Metatarsal4, M5= Metatarsal5, MF=Midfoot, $\mathrm{MH}=$ Medialheel, $\mathrm{LH}=$ Lateralheel. 